\title{
Screening, brief intervention, and referral to treatment among homeless and marginally housed primary-care patients in Skid Row
}

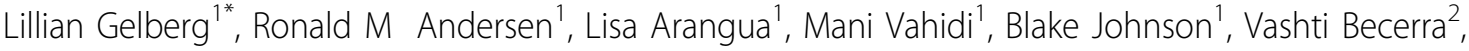 \\ Colleen Duro ${ }^{2}$, Steve Shoptaw ${ }^{2}$
}

From International Network on Brief Interventions for Alcohol Problems (INEBRIA) Meeting 2011

Boston, MA, USA. 21-23 September 2011

The University of California at Los Angeles Quit Using Drugs Intervention Trial (UCLA QUIT) tested a very brief primary-care-based screening and brief intervention (SBI) approach to reduce risky substance use and substance-related harm in safety-net clinics. The QUIT involves screening, very brief clinician advice (two to three minutes), and two telephone drug-use health education sessions versus usual care (control group) $(n=240$ per condition). We present findings on unique recruitment issues in Skid Row, an east-central area of Los Angeles with a high population of homeless individuals. Between February 18 and April 28, 2011, previsit screening of adults in the clinic waiting room was conducted using a touch screen tablet PC. At-risk substance use was defined as casual, frequent, or heavy episodic use without the physiological or psychological manifestations of dependence (i.e., a score of 4 to 26 on the World Health Organization's Alcohol, Smoking, and Substance Use Involvement Screening Test [ASSIST]). The focus of the study was on risky stimulant use, however, patients were screened for co-occurring alcohol, tobacco, and other drug use. A total of 920 patients were approached: $89 \%$ were $\geq 40$ years old; $68 \%$ were male; and $62 \%$ were black. Of these, 706 were excluded prior to taking the ASSIST (reasons included being pregnant, presenting for a nonprimary-care visit, being in substance use treatment, or refusal to participate). Of the 214 patients who completed the ASSIST, substance use rates based on scores were as follows: no/low risk, $11 \%$; moderate risk, $42 \%$; and dependence, $47 \%$. Totals for each risk group, respectively, were as follows: tobacco $(55,101,58)$, alcohol $(62,98,54)$, cannabis $(94,77,43)$, cocaine $(89,74,51)$, amphetamines $(145,45,23)$, inhalants $(185,20,9)$, sedatives $(143,45$, $26)$, hallucinogens $(174,30,10)$, and opioids $(130,54,30)$. Few patients qualified for the study because of substance use treatment or co-occurring alcohol or cannabis dependence. Key informants revealed that many of those approached received intermittent substance use treatment required by shelters. Enrollment criteria were relaxed to allow intermittent past-month substance use treatment or co-occurring alcohol or cannabis dependence. Enrollment rates increased several-fold. Our findings indicate SBIRT conducted in clinics with homeless and marginally housed populations must be tailored to their unique substance use and housing characteristics.

\section{Author details \\ 'UCLA Department of Family Medicine \& School of Public Health, Los Angeles, CA, USA. ${ }^{2}$ UCLA Department of Psychology \& Education, Los} Angeles, CA, USA.

Published: 9 October 2012

doi:10.1186/1940-0640-7-S1-A58

Cite this article as: Gelberg et al.: Screening, brief intervention, and referral to treatment among homeless and marginally housed primarycare patients in Skid Row. Addiction Science \& Clinical Practice 2012 7(Suppl 1):A58. 